\title{
Incorporation of directional effects of the retina into computations of optical transfer functions of human eyes
}

\author{
Pablo Artal \\ Instituto de Optica, Consejo Superior de Investigaciones Científicas, Serrano 121, 28006 Madrid, Spain
}

Received November 21, 1988; accepted July 18, 1989

\begin{abstract}
Two-dimensional optical transfer functions (OTF's) of individual human eyes are computed from the retinal images of a point test object, using a phase-retrieval method. The retinal reflection directionality effect is included in the computations by means of an apodization pupil, and subsequently the Stiles-Crawford effect is also considered. The modulation transfer functions obtained when the retinal reflection directionality effect is considered show lower values of the modulation; on the other hand, their two-dimensional form and the corresponding phase transfer functions remain practically unchanged. The importance of the Stiles-Crawford apodization depends on the wave aberration of the individual subject, but in general it produces an improvement in image quality, and the modulation transfer function becomes more symmetrical.
\end{abstract}

\section{INTRODUCTION}

The double-pass method has been used widely for objective determinations of image quality in the human eye. The results were usually expressed in terms of the one-dimensional modulation transfer function (MTF) computed from aerial retinal images of line test objects. ${ }^{1-3}$ However, because of the irregularities of the retina and the asymmetries of the wave aberration of the eye, the use of a point as test object gives more complete information. ${ }^{4}$ A hybrid opticaldigital method for the determination of the retinal image of a point test [i.e., the point-spread function (PSF) of the individual human eye] was developed by Santamaría et al. ${ }^{5}$ The method allows us to obtain the corresponding twodimensional optical transfer function (OTF). A subsequently presented phase-retrieval method ${ }^{6}$ permits the determination of the wave aberration of human eyes from the PSF results.

Several authors have pointed out that the image-quality results obtained by using the double-pass method cannot be completely accurate because of a variety of uncontrolled difficulties. Berny ${ }^{7}$ evaluated the importance of coherence properties in the retinal reflection; Gorrand ${ }^{8}$ analyzed the influence of the image degradation caused by retinal scattering; and Gorrand et al. ${ }^{9,10}$ studied the retinal reflection directionality effects. This physical effect is due to the waveguide structure of the cone receptors; its variation with the position of the entering beam in the pupil was measured by van Blockland and van Norren. ${ }^{11}$ The scattering diagrams for this effect show approximately the same functional dependence as that for the Stiles-Crawford effect. The reflectivity of the retina has approximately a Gaussian dependence on the position of the incident beam in the pupil plane. On the basis of these results, Deeley and Drasdo ${ }^{12}$ suggested that MTF data obtained by the double-pass method $^{3}$ would be lower than actual MTF.

The Stiles-Crawford effect has the same physical cause as the directionality in the reflection. However, since it is a postdetection effect, it has been measured by psychophysical methods. Stiles-Crawford apodization has been incor- porated into MTF calculations by application of several theoretical models, ${ }^{13,14}$ but so far as I know it has not been introduced into any computation for the data of actual individual eyes. This is an important point, because it has been shown that, in general, the effect of apodization filters depends on the intrinsic image quality of the optical system considered. Moreover, this apodization is closely related to the retinal reflection directionality. In fact, Deeley and Drasdo ${ }^{12}$ did not take the Stiles-Crawford effect into account in their study; they assumed that it would be compensated for by the nonincluded retinal reflection directionality effects. However, until now it has not been thought possible to consider both effects simultaneously in a correct way.

In this paper two-dimensional OTF's are computed from aerial retinal images of a point test object by using a phaseretrieval method by the incorporation of a simplified model of the retinal reflection directionality effects. Subsequently, the Stiles-Crawford effect is also included in the computations of OTF's from wave aberration data. In what follows, the computational method, OTF results, and a general discussion are presented.

\section{COMPUTATIONAL METHOD FOR OPTICAL TRANSFER FUNCTION DETERMINATION}

The aerial retinal image of a point test is computed by adding short-term images obtained using the optical-digital procedure presented previously. ${ }^{5}$ All the experimental results of retinal images were obtained in foveal vision with an incident light $632 \mathrm{~nm}$ in wavelength. When the retinal directional effects are not considered, the PSF and the twodimensional OTF $\{H(u, v)=M(u, v) \exp [i O(u, v)]$, where $u$ and $v$ are spatial frequencies in the $x$ and $y$ directions\} are obtained by direct deconvolution of the aerial retinal image. The wave aberration, $W(\alpha, \beta)$, where $\alpha$ and $\beta$ are normalized pupil coordinates, is then computed from the actual PSF result and the modulus of the constant pupil transmission function, $p(\alpha, \beta)$, by means of a phase-retrieval method. ${ }^{6}$ The retinal effects are included in the computations through 
an apodized pupil transmission function. Despite the complexity of foveal reflection, here its spatial properties are described by a simplified model.

However, there are other factors with influence on the retinal reflection that are not considered: for instance, polarization and bleaching of the visual pigments. The directionality of the retinal reflection is a physical effect of the waveguide structure of the retinal cones. It was measured by analyzing scattering diagrams of an incident beam in the fovea. ${ }^{11}$ The Stiles-Crawford effect was measured by psychophysical means. The results for both effects, despite individual differences among subjects, show approximately the same functional dependence. ${ }^{15}$ For the sake of simplicity, in what follows, both effects are considered by means of the modulus of an apodized pupil function, $p_{d}(\alpha, \beta)$, that is expressed as a Gaussian function with the same bandwidth given by ${ }^{13}$

$$
\begin{aligned}
p_{d}(\alpha, \beta) & =\exp \left(-0.108 R^{2} r^{2}\right) & & \text { if } \alpha^{2}+\beta^{2}<1 \\
& =0 & & \text { if } \alpha^{2}+\beta^{2} \geq 1,
\end{aligned}
$$

where $r^{2}=\alpha^{2}+\beta^{2}$ and $R$ is the pupil radius. The retinal reflection directionality is included in the computations by means of the apodized pupil described above. The wave aberration with the effect of the reflection directionality, $W_{d}(\alpha, \beta)$, is retrieved from the PSF data and the apodization pupil $p_{d}(\alpha, \beta)$. The wave aberration is obtained by means of a retrieval procedure ${ }^{6}$ with a final mean-square error in the algorithm that is less than 0.05 for all the considered subjects. The modified OTF including the reflection directional effects, $H_{d}(u, v)=M_{d}(u, v) \exp \left(i O_{d}(u, v)\right.$, is then computed as a normalized autocorrelation of the complex pupil function

$H_{d}(u, v)=\left\{p(\alpha, \beta) \exp \left[i W_{d}(\alpha, \beta)\right]\right\} *\left\{p(\alpha, \beta) \exp \left[i W_{d}(\alpha, \beta)\right]\right\}$,

where $*$ means auto correlation.

In the whole procedure for the determination of the OTF with the directional influence, the apodization effect has been included twice, whereas in fact during the double-pass measurements the apodization affects only the second pass (i.e., after retinal reflection). Under this condition, the resulting OTF, $H_{d}(u, v)$, obtained by using this procedure is a lower limit because the directional effect has been overweighted in the calculations. The actual OTF will be between $H(u, v)$ and $H_{d}(u, v)$. Finally, the Stiles-Crawford effect is included in the computations of the OTF, $H_{s}(u, v)$, through the wave aberration $W_{d}(\alpha, \beta)$ by means of a normalized autocorrelation of the apodized complex pupil function,

$H_{s}(u, v)=\left\{p_{d}(\alpha, \beta) \exp \left[i W_{d}(\alpha, \beta)\right]\right\} *\left\{p_{d}(\alpha, \beta) \exp \left[i W_{d}(\alpha, \beta)\right]\right\}$.

\section{RESULTS AND DISCUSSION}

Figure 1 shows the two-dimensional OTF $H(u, v)$ obtained without consideration of directionalities in the retinal reflection for an emmetropic subject with a pupil diameter of $5 \mathrm{~mm}$. Figure 1(a) shows a contour plot, at 0.1 intervals, of the twodimensional MTF, $M(u, v)$, and Fig. 1(b) shows a contour plot, at 10-deg intervals, of the phase transfer function (PTF) $O(u, v)$. In a recent study, Artal et al..$^{16}$ analyzed the influence of the PTF on the image-quality determinations in hu-

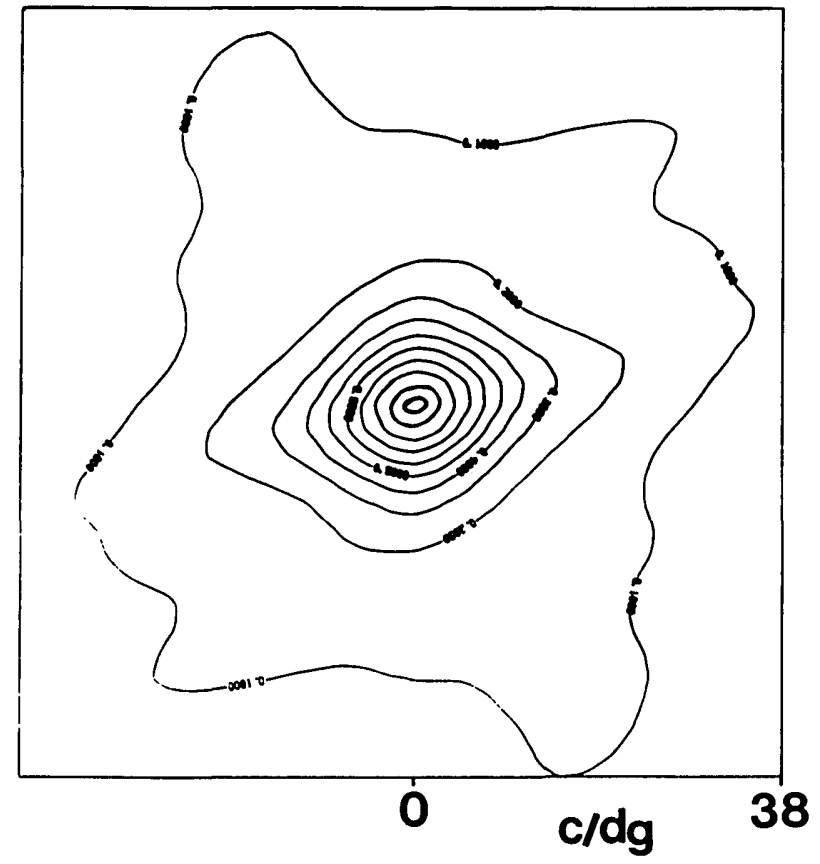

(a)

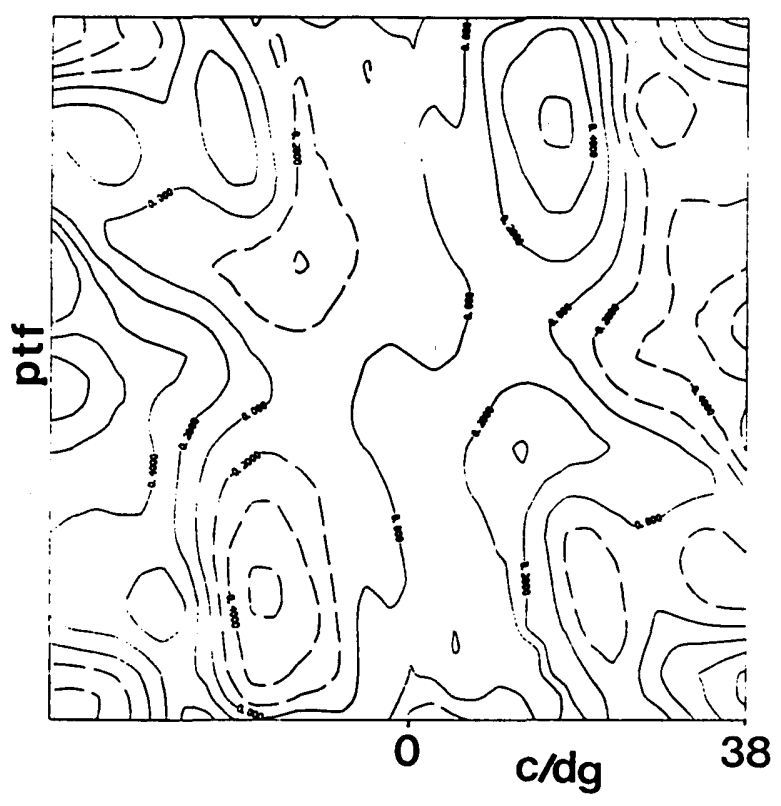

(b)

Fig. 1. (a) Contour plot at 0.1 intervals, of the two-dimensional MTF, $M(u, v)$, of an emmetropized subject with a 5-mm pupil diameter. Results were obtained in foveal vision, with monochromatic incident light $(632 \mathrm{~nm})$ without consideration of retinal directionalities in the calculations. (b) Contour plot at 10-deg intervals of the two-dimensional phase transfer function (PTF), $O(u, v)$, for the same subject and conditions as in Fig. 1(a). c/dg, Cycles per degree.

man eyes. They showed that, even in the case of an emmetropic subject, the PTF has an important effect, with nonzero values at significant spatial frequencies. Its main significance is in relation to the asymmetries present in the eye's optical system. Santamaría et al. ${ }^{5}$ and Artal et al. ${ }^{6}$ showed the importance of asymmetric aberration in the image quality of human eyes. Those results were in good agreement with 
previous work by Walsh and co-workers ${ }^{17,18}$ and Howland and Howland. 19

The two-dimensional OTF, $H_{d}(u, v)$ is shown as a MTF in Fig. 2(a) and as a PTF in Fig. 2(b) for the same subject as in Fig. 1; the apodization pupil that simulates the retinal reflection directionality is included in the calculations for these plots. As is noted above, in the double-pass imaging process only the second pass is affected by the apodization, because of the directionality in the reflection. Therefore, we obtain a

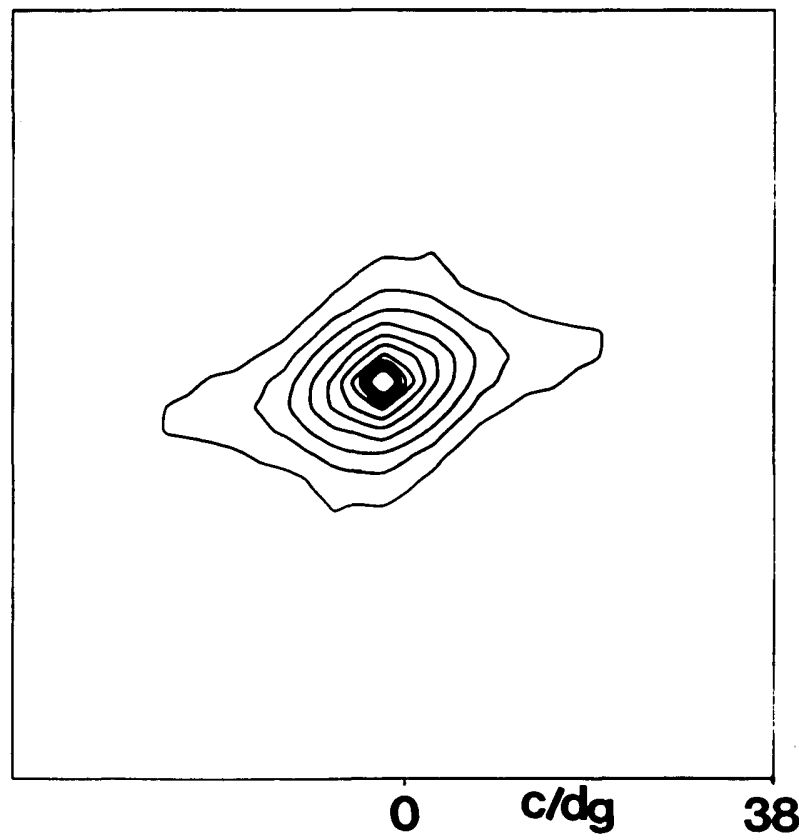

(a)

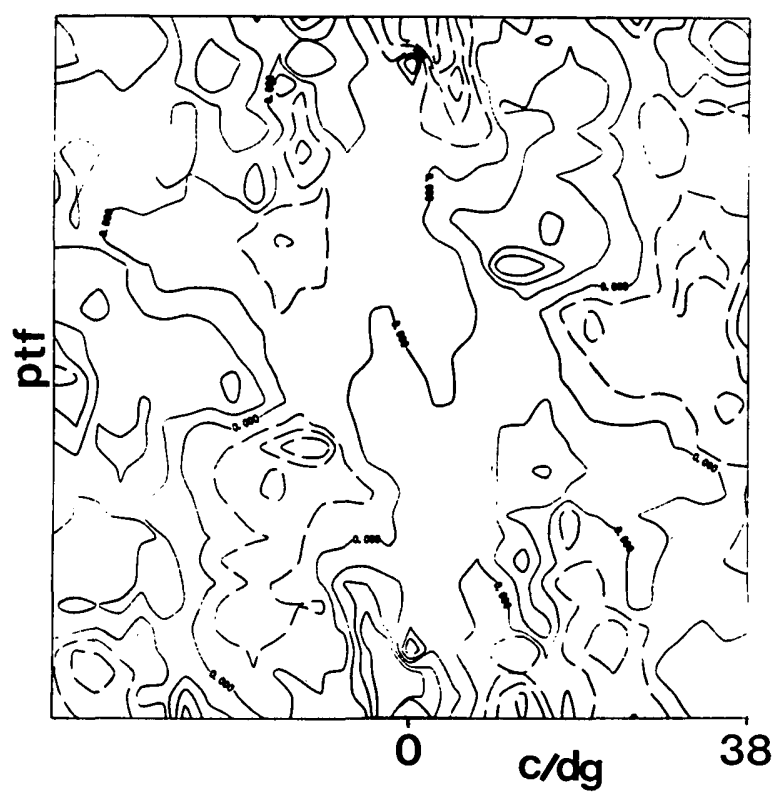

(b)

Fig. 2. (a) Contour plot at 0.1 intervals of the two-dimensional MTF, $M_{d}(u, v)$, obtained when the directionality of the retinal reflection is included in the computations, for the same subject as in Fig. 1. (b) Contour plot at 10-deg intervals of the two-dimensional PTF with consideration of retinal reflection directionality, $O_{d}(u, v)$, for the same subject and conditions as in (a). c/dg, Cycles per degree.

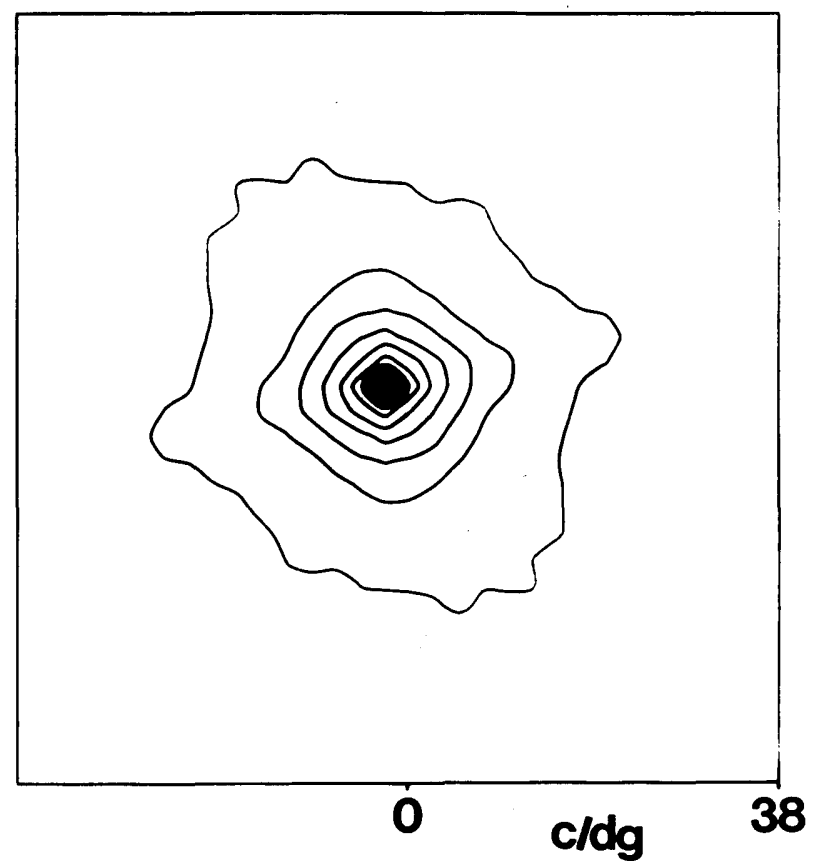

Fig. 3. Contour plot at 0.1 intervals of the two-dimensional MTF, $M_{s}(u, v)$, with the incorporation of the Stiles-Crawford effect after the consideration of retinal reflection directionality. c/dg, Cycles per degree.

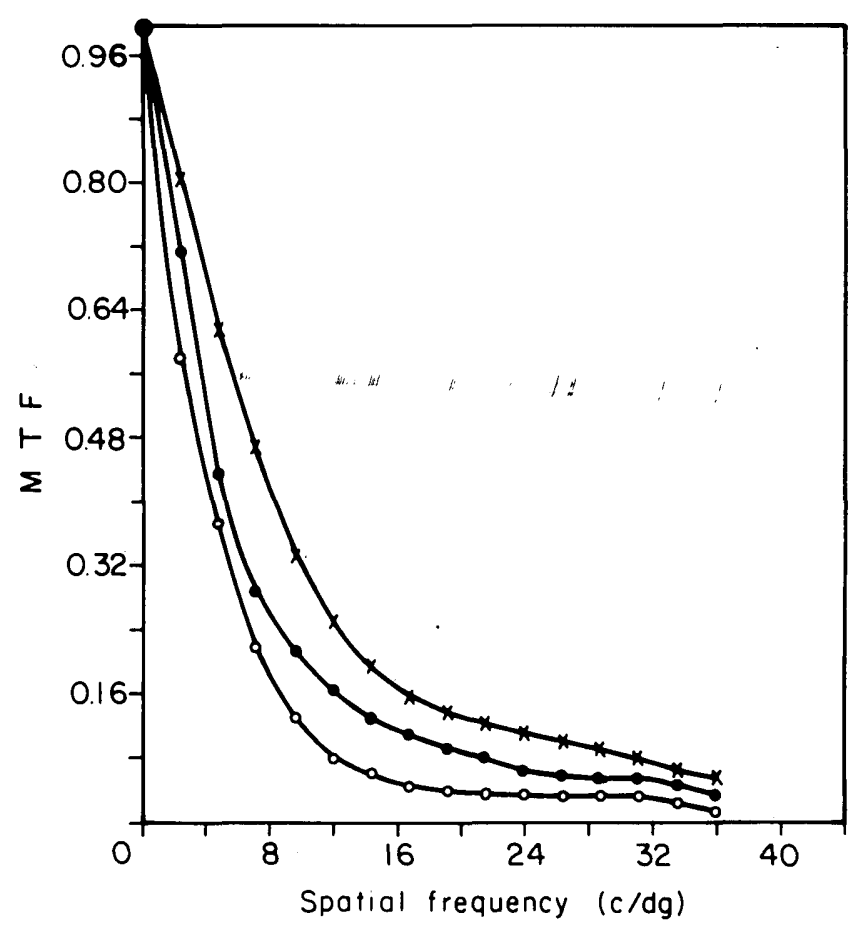

Fig. 4. Comparison of sections of the MTF's at 0 deg: $\times, M(u, v)$ with no consideration of directional effects [Fig. 1(a)]; $\mathrm{O}, M_{d}(u, v)$ with the retinal reflection directionality incorporated into the computations [Fig. 2(a)]; $\bullet, M_{s}(u, v)$ also with incorporation of the Stiles-Crawford effect (Fig. 3). c/dg, Cycles per degree.

lower limit for the OTF. The new results show approximately the same asymmetric shape but with lower values of modulation. Both PTF's are similar [compare Figs. 1(b) and 2(b)], except for the noise that has been introduced in the computations. The MTF $M_{s}(u, v)$ with allowance for the Stiles- 
Crawford effect is shown in Fig. 3. The results appear to be slightly more symmetrical in comparison with the MTF before detection [Figs. 1(a) and 2(a)], and the values of the modulation are higher than in the predetection MTF, $M(u$, $v)$. All the calculations have been done for several emmetropized subjects, and it has been shown that the relative importance of the Stiles-Crawford effect depends on the wave aberration that is present in the eye of each subject. In general, the image quality is improved, and the two-dimensional MTF is made more symmetrical, especially at intermediate spatial frequencies. In order to evaluate the relation between the effect of Stiles-Crawford apodization on image quality and the pupil size, the relative Strehl ratio, i.e., the ratio of the maximum illuminance in the retinal PSF after the apodization to that before apodization, has been calculated for different subjects and pupil sizes. The mean results for five subjects show a larger relative improvement in the image quality for smaller pupil diameters. Finally, Fig. 4 shows a comparison of sections of the MTF at 0 deg without consideration of directional effects $[M(u, v)]$, with the incorporation of the retinal reflection directionality $\left[M_{d}(u, v)\right]$, and with consideration of the Stiles-Crawford effect.

\section{ACKNOWLEDGMENTS}

I thank Javier Santamaría, Julian Bescós, and Rafael Navarro for their helpful suggestions while the research was in progress; Ana Plaza and Manuel Perez for their careful technical collaboration; and F. W. Campbell, Cambridge University (UK), for many helpful discussions during my summer visit to his laboratory. This research was supported partially by the Comision Interministerial de Ciencia y Tecnologia Spain.

\section{REFERENCES}

1. M. F. Flamant, "Etude de la repartition de lumiere dans l'image retinienne d'une fente," Rev. Opt. 34, 433-459 (1955).

2. J. Krauskopf, "Light distribution in human retinal images," J. Opt. Soc. Am. 52, 1046-1050 (1962).

3. F. W. Campbell and R. W. Gubisch, "Optical performance of the human eye," J. Physiol. (London) 186, 558-578 (1966).
4. A. Arnulf, J. Santamaría, and J. Bescós, "A cinematographicmethod for the dynamic study of the image formation by the human eye. Microfluctuations of the accommodation," J. Opt. 12, 123-128 (1981).

5. J. Santamaría, P. Artal, and J. Bescós, "Determination of the point-spread function of human eyes using a hybrid opticaldigital method," J. Opt. Soc. Am. A 4, 1109-1114 (1987).

6. P. Artal, J. Santamaría, and J. Bescós, "Retrieval of wave aberration of human eyes from actual point-spread-function data," J. Opt. Soc. Am. A 5, 1201-1206 (1988).

7. F. Berny, "Correlation de phase entre deux sources formees sur un surface diffusante. Application a la retine humaine," Vision Res. 12, 1631-1645 (1972).

8. J. M. Gorrand, "Diffusion of the human retina and quality of the optics of the eye on the fovea and peripheral retina," Vision Res. 19, 907-912 (1979).

9. J.M. Gorrand, R. Alfieri, and J. Y. Boire, "Diffusion of the retinal layers of the living human eyes," Vision Res. 24, 1097-1106 (1984).

10. J. M. Gorrand, "Reflection characteristics of the human fovea assessed by reflecto-modulometry," Ophthalmol. Physiol. Opt. 9, 53-60 (1989).

11. G. L. van Blockland and D. van Norren, "Intensity and polarization of light scattered at small angles from the human fovea," Vision Res. 26, 485-494 (1986).

12. R. J. Deeley and N. Drasdo, "The effect of optical degradation on the contrast sensitivity function measured at the fovea and in the periphery," Vision Res. 27, 1179-1186 (1987).

13. A. van Meeteren, "Calculations on the optical modulation function of the human eye for white light," Opt. Acta 21, 395-412 (1972).

14. R. Navarro, J. Santamaría, and J. Bescôs, "Accommodationdependent model of the human eye with aspherics," J. Opt. Soc. Am. A 2, 1273-1281 (1985).

15. G. L. van Blockland, "Directionality and alignment of the foveal receptors, assessed with light scattered from the human fundus in vivo," Vision Res. 26, 1791-1795 (1988).

16. P. Artal, J. Santamaría, and J. Bescós, "Phase transfer function of human eyes and its influence on the point-spread function and wave aberration," J. Opt. Soc. Am. A 5, 1791-1795 (1988).

17. G. Walsh, W. N. Charman, and H. C. Howland, "Objective technique for the determination of monochromatic aberration of the human eye," J. Opt. Soc. Am. A 1, 987-992 (1984).

18. G. Walsh and W. N. Charman, "Measure of the axial wavefront aberration of the human eye," Ophthalmol. Physiol. Opt. 5, 2331 (1985).

19. H. C. Howland and B. Howland, "A subjective method for the measurement of monochromatic aberration of the human eye,"J. Opt. Soc. Am. 67, 1508-1518 (1977). 